\title{
Emotional Consequences of Persistently Elevated PSA with Negative Prostate Biopsies
}

\author{
John G. Scott ${ }^{1,2,}$, Eric K. Shaw ${ }^{5}$ Asia Friedman ${ }^{4}$, Jeanne M. Ferrante ${ }^{1,3}$ \\ ${ }^{1}$ Department of Family Medicine and Community Health, Robert Wood Johnson Medical School, University of Medicine and \\ Dentistry of New Jersey, New Brunswick, USA \\ ${ }^{2}$ Corner Medical, Northeastern Vermont Regional Hospital, Lyndonville, USA \\ ${ }^{3}$ Cancer Institute of New Jersey, New Brunswick, USA \\ ${ }^{4}$ Department of Sociology and Criminal Justice, University of Delaware, Newark, USA \\ ${ }^{5}$ Savannah Campus, Mercer University School of Medicine, Savannah, USA \\ *Corresponding author: j.scott@nvrh.org
}

Received January 13, 2013; Revised February 04, 2013; Accepted February 25, 2013

\begin{abstract}
False positive results are among the most common adverse outcomes of cancer screening tests. Up to $75 \%$ of men with an elevated prostate specific antigen (PSA) have had a negative prostate biopsy. Adverse emotional effects of false positive prostate cancer screening have been documented using surveys, but there is nothing in this literature describing the lived experience of having a false positive PSA. In a qualitative interview study of sixteen men who had false positive PSAs, we found that all subjects experienced transient anxiety while waiting for the results of their prostate biopsies. In addition there were three persistent emotional responses: (1) increased fear of cancer; (2) relief with increased vigilance; (3) relief with less worry about elevated PSA. The attitude and recommendations of urologists influenced the emotional responses. Some men expressed frustration with the PSA as a screening test. Many men had multiple negative prostate biopsies. These data suggest that there should be more effort to develop guidelines for subsequent prostate biopsies after an initial negative biopsy, and more effort to encourage primary care clinicians and urologists to discuss emotional risks of PSA testing with their patients.
\end{abstract}

Keywords: prostate cancer, prostate specific antigen, cancer screening, false positive screening tests, qualitative research

\section{Introduction}

Among the most common adverse outcomes of cancer screening tests arefalse positive results. Croswell, et al., for example, found in a multimodal cancer screening trial that the cumulative risk of at least one false positive result after the 14 recommended screening tests was $60.4 \%$ [1].In the European randomized study of screening for prostate cancer, Kilpelainen et al. found that $20 \%$ of participants had at least one false positive prostate specific antigen (PSA)(defined as an elevated PSA that results in one or more negative prostate biopsies, with no diagnosis of prostate cancer made in one year) [2] and in the same study, Schroeder et al found that $75 \%$ of men with an elevated PSA had a negative prostate biopsy [3]. False positive PSAs can result from a number of causes other than prostate cancer, including benign prostatic hypertrophy, prostatitis, bladder infection, bicycle or horseback riding, instrumentation such as cystoscopy or colonoscopy and certain medications [4]. Consequences of false positive cancer screening tests include unnecessary invasive procedures [1], increased utilization of health care resources $[5,6]$, and decreased adherence to further screening $[7,8,9,10]$.
Adverse emotional effects have also been reported in patients who have false positive cancer screening results $[5,8,11,21]$. False positive mammograms have been the best studied in this regard [5,11,12,16,18,20,21]. A recent meta-analysis of these studies found that women who had false positive mammograms had greater anxiety and distress about breast cancer, more frequent breast selfexams, higher perceived effectiveness of screening mammography, and more generalized anxiety than women who had normal mammograms [20]. Van der Steeg, et al. found that women had reduced quality of life and feelings of anxiety that lasted for at least a year after a false positive mammogram [21].

Adverse emotional consequences in men who have false positive prostate cancer screening results have been less well studied $[15,17,22,23]$. Macefield et al. found that although most men coped well with a negative prostate biopsy, a significant percentage of men had high distress and tense anxious mood that lasted for at least 12 weeks after the biopsy [22]. All of these studies utilized surveys to assess emotional and emotional consequences and therefore could not capture the lived experience of having a false positive PSA. Furthermore, the longest follow-up in any of these survey studies was one year [24], and most were at 6-12 weeks after the negative biopsy $[17,22,23]$. 
In this study, we used qualitative methods in order to obtain rich descriptions of the long-term emotional consequences for men who had an elevated PSA and one or more negative prostate biopsies. For purposes of this paper the word "emotional" refers to descriptions of how men described their feelings and does not assume any particular emotional states. The interviews with the subjects were recorded from one to twenty four years after the initial negative biopsy.

\section{Methods}

\subsection{Study Sample}

The data reported here are from semi-structured indepth interviews that were part of a larger study that was conducted between March 2009 and May 2010 to qualitatively explore factors influencing men's decisions regarding prostate cancer screening and treatment among men who were never screened or those who had an abnormal screening [25]. For the larger study, a purposive sample of men $(\mathrm{N}=64)$ ages 50 and older was recruited via flyers posted in community-based organizations and via newspaper advertisements in northern New Jersey. Our inclusion criteria included men age 50 and older who were never screened for prostate cancer or who had an abnormal screening and, subsequently, had a normal biopsy or cancer diagnosis. Men were excluded if they did not speak English or Spanish. In some cases, men's wives also participated in the interview. The study protocol was approved by the Institutional Review Boards of University of Medicine and Dentistry of New Jersey, Cancer Institute of New Jersey, and U.S. Army Medical Research and Materiel Command. All participants provided informed consent and received $\$ 30$ cash for completing the interview.

\subsection{Data Collection}

In-depth interviews were conducted in English or Spanish by four qualitative interviewers in the men's homes, the offices of the community-based organizations, or at another convenient location agreed upon by the interviewer and participant. The research team developed an interview guide using Andersen's Behavior Model of Health Services Use [25] as the conceptual framework. Participants were asked open ended questions about prostate cancer and screening for prostate cancer, feelings about prostate cancer screening, sources of information, any discussions with doctors regarding prostate cancer screening, feelings toward health and healthcare in general, experiences with other family members or friends with cancer, personal health practices, and use of health services. Patients with abnormal screening tests were asked to describe their feelings about the experience of prostate biopsy and of treatment if the biopsy was positive. Interviews lasted 30-90 minutes and were digitally recorded and transcribed verbatim. Recruitment and interviews continued until data reached saturation $(\mathrm{N}=64)$.

The data sample for this paper is a subset of the interviews for the larger study [26]. The inclusion criteria for this subset were a positive screening test and one or more negative prostate biopsies $(\mathrm{N}=16)$. Interview audiofiles, transcripts, and interviewer fieldnotes served as data sources for our analysis. Transcripts were deidentified and imported into ATLAS.ti (Atlas.ti Scientific Software Development GmbH, Berlin, Germany) for coding and analysis.

\subsection{Data Analysis}

The analysis team consisted of a family physician with 25 years experience in private practice (JS), an academic family physician with extensive research experience (JF), and two sociologists (ES, AF).

Our qualitative analysis used a grounded theory approach that involved a series of immersion/crystallization cycles [27]. This involved immersing ourselves in the data through iterative cycles of readings and reflections. This immersion process creates intimate familiarity with the data so that the underlying structure of the data begin to become apparent and insights and themes crystallize through discussion among the analysis team. Initially, the research team read transcripts together to understand the content and to develop a set of preliminary codes. Group analysis continued until consensus was reached regarding coding schemes. We then independently analyzed the remaining data, meeting regularly to discuss interpretations and to refine coding schemes as needed. All transcripts were independently coded by at least two research team members, and any differences in coding were resolved through group consensus. Next, data within codes were reread and analyzed in a second immersion/crystallization cycle, and emerging themes and interpretations were identified. The emotional consequences of false positive prostate cancer screening were central to this analytic cycle. A third immersion/crystallization cycle was used to identify negative or disconfirming evidence for our themes. Through this process, themes and interpretations were refined. The quotations included in this paper represent and illustrate our key findings.

\section{Results}

Subjects were all Caucasian with a relatively high education level, with ages ranging from 51-83. We did not systematically gather PSA values that triggered biopsy, but a number of subjects reported their PSA values in the interviews. Table 1 shows the demographic characteristics of the subjects as well as PSA values when available, number of prostate biopsies, and number of years since the initial biopsy.

All of our subjects experienced transient anxiety while waiting for the results of their prostate biopsies. In addition, we identified three types of persistent emotional responses to false positive prostate cancer screens: Increased fear of cancer; relief with increased vigilance; relief with less worry about elevated PSA (see Table 2).In addition, two subjects expressed frustration over the uncertainties and limitations of the PSA as a marker for early prostate cancer. Not surprisingly, the attitude and recommendation of the urologist was influential in how subjects responded to false positive screening tests. We discuss each of these responses below and illustrate them with representative excerpts from the interviews.

\subsection{Initial Anxiety}


All subjects experienced significant anxiety while waiting for the results of their prostate biopsies. This anxiety occurred with every biopsy, not just the first one. For example, one patient said,

"Well I think it was scary to say hey, if this test comes back and I have prostate cancer..., you think of cancer as, you're gonna die, you know? So, it was scary from that point of view."

Table 1. Sample demographics

\begin{tabular}{|c|c|c|c|c|c|}
\hline Age & Ethnicity & education & PSA & \# Biopsies & $\begin{array}{c}\text { Years Since } \\
1^{\text {st }} \text { Biopsy }\end{array}$ \\
\hline 51 & Caucasian & college & $*$ & 1 & $*$ \\
\hline 52 & Caucasian & college & 8.52 & 3 & 5 \\
\hline 58 & Caucasian & college & $*$ & 1 & 4 \\
\hline 58 & Caucasian & college & 12 & 9 & 9 \\
\hline 61 & Caucasian & college & $*$ & 1 & 1.5 \\
\hline 62 & Caucasian & college & 4.1 & 7 & 13 \\
\hline 62 & Caucasian & college & 7.5 & 1 & 1 \\
\hline 64 & Caucasian & college & 3.6 & 1 & 4 \\
\hline 65 & Caucasian & college & 4 & 3 & 10 \\
\hline 65 & Caucasian & college & 8 & 1 & 5 \\
\hline 65 & Caucasian & college & 6 & 2 & 1 \\
\hline 66 & Caucasian & $*$ & 6 & 2 & 9 \\
\hline 66 & Caucasian & college & 8 & 1 & 7 \\
\hline 73 & Caucasian & college & 4.6 & 8 & 10 \\
\hline 79 & Caucasian & college & 15 & 1 & 6 \\
\hline 83 & Caucasian & college & 18 & 5 & 24 \\
\hline$*$
\end{tabular}

* Missing data

Table 2. Theme Frequencies

\begin{tabular}{|c|c|}
\hline Theme & Number of Subjects \\
\hline Increased Fear of Cancer & 5 \\
\hline Relief Followed by Increased Vigilance & 7 \\
\hline $\begin{array}{c}\text { Relief Followed by Less Worry About } \\
\text { Elevated PSAs }\end{array}$ & 4 \\
\hline
\end{tabular}

\subsection{Increased Fear of Cancer}

One prominent theme from our analysis was increased fear of developing prostate cancer. One subject, who had nine negative prostate biopsies, even wanted a prophylactic prostatectomy so that he would not have to worry about getting prostate cancer. He said,

"Can we just take out the prostate itself? Is it good for me anymore? It seems to be nothing but a problem here. It's causing me to go through this every six months."

Another subject felt that he would eventually get prostate cancer, even though he had a negative biopsy:

"Yeah, in my lifetime, I'm very concerned that I may face problems with my prostate. I believe that the longer any of us live, the more likelihood that we're going to be afflicted with some type of cancer. So, yes, that is my concern."

The urologist often amplified the patient's increased fear of cancer by emphasizing the lack of certainty of a negative biopsy. One patient said, for example,

"So by doing the biopsy; that eliminated the cancer aspect of it...at least at that time. I think he did 10 needle punches or something...5 going up and 5 coming down? And I did that 2 or 3 times so even in his own mind he probably said; okay I did one biopsy...came out negative and then maybe a year later he wanted to do it again because PSA...you know, you're taking the needle biopsy in different places and maybe you didn't hit the right places."

\subsection{Relief Followed by Increased Vigilance}

Some subjects were relieved that their prostate biopsies were negative, but responded with increased vigilance and increased frequency of prostate cancer screening, ranging from every four months to once a year. One such subject said,

"So you gotta' continue, monitoring it. Taking a look at it. So that's what motivated (me) to think about, to do that. And I feel a little--I feel safer, I feel more secure about things, and just to continue life the way things have been goin,",

\subsection{Relief Followed by Less Worry about Elevated PSAs}

Some subjects were relieved that their prostate biopsies were negative and became much less concerned with their persistently elevated PSAs. One subject in this category had a PSA as high as 18 with five negative prostate biopsies. He said,

"I had accepted the fact that at least for me and possibly for others you were going to get skewed results. That's just the way it was with me and I just determined that what I should not do was be petrified if I had a high PSA... and I shouldn't let the scare of prostate cancer petrify me as it might have done before that."

Some urologists reportedly reassured patients about their persistently elevated PSA after several negative biopsies. One of these patients said,

"So again he [the urologist] did a biopsy, found nothing, and at a certain point we both decided-and he was the one who really said that I'm one of those where the number has very little meaning-so he continued having me take the blood tests-the PSA-but he said unless there's a major jump, he wouldn't do anything. "

\subsection{Frustration with Uncertainties of PSA}

Two subjects, one in the increased fear of cancer group, and one in the relief with less worry about elevated PSA group, expressed marked dissatisfaction with PSA as a screening test. The patient who had an increased fear of cancer said,

"So I'm interested because I'm interested in my health and the PSA is a crazy test I found out, it has nothing to do with a $100 \%$ reality, which is disturbing to me, and probably disturbing to the health community."

The other patient said,

"And so what I've been reading lately is that there's a lot of false positives with a PSA test. In fact, I think I've read in some publication that it makes you almost believe, 'What's the point of having a PSA test if 50\% of the time it's correct and 50\% of the time it's wrong?' Nothing more than causing you to go see a urologist. And then of course I've read accounts where even if you do have prostate cancer at a certain age, it's not gonna eventually kill you...So I'll go back to the urologist next month, because he already wrote me the prescription and everything, just to find out what my PSA reading is going to be, but if it's, say, in the range of, like I said, 5.5 when the high was 7.5 when he did the last biopsy that came back negative, and if he suggests another round of biopsies, I may question him on that. I may say, 'Thanks, but no thanks.", 


\section{Discussion}

While survey studies have shown short-term emotional effects of false positive PSAs, our subjects reported significant emotional consequences that persisted for up to 24 years from the initial biopsy (see Table 1). For most of the men in this study, persistently elevated PSA led to a cascade of biopsies and further PSA tests, sometimes as often as every four to six months. One patient (quoted in the results section) had nine negative prostate biopsies, while seven subjects had a single biopsy. As pointed out in the introduction, the false positive rate for PSA screening approaches 75\%.[3]The financial cost of false positive PSA screening must therefore be enormous. In addition to the financial costs, men in all three groups experienced significant emotional distress before they received the results of their biopsies. The most common persistent long-term emotional responses were increased fear of cancer and relief but increased vigilance, both of which resulted in increased frequency of screening and often subsequent biopsies. Even the small group of men who felt reassured about their persistently elevated PSA only did so after multiple negative biopsies. Two of the subjects, despite having multiple PSA tests, and multiple biopsies, had come to distrust the value of PSA screening.

It is important to note that there seemed to be no consensus among the urologists about how many biopsies are necessary with a persistently elevated PSA. Some patients had a single biopsy, while most had two or more. It would seem that, given the emotional and financial cost of false positive PSAs, evidence-based guidelines about further testing after a negative prostate biopsy are sorely needed [28].

In a previous analysis of the complete dataset from this study, we noted that no patients reported that any of their physicians discussed risks of PSA screening [26]. This is an important omission, particularly given the recent finding by the U.S. Preventive Services Task Force that the risks of PSA screening outweigh the potential benefits [29].

We recognize that this study has several limitations. Although the study was small by quantitative standards, we used standard qualitative methods for determining an adequate sample size; that is, we continued to interview subjects and do iterative analysis until we were hearing no new themes in the interviews. Since this subset of the study sample was limited to highly educated Caucasian males in one region of the country, emotional responses to false negative PSA screening might be different in other ethnic groups, men with less education, and/or other regions of the US. Our interpretation of these data is of necessity influenced by our life experience. Others might view the data differently and come to different conclusions. The validity of our analysis, however, is enhanced by the diversity of training and experience of the analysis team and by our use of reflexivity (reflecting on our own experiences).

\section{Conclusions}

Rich descriptions of men's experiences of false positive PSA screening reveal that men experience significant emotional distress, which is often followed by increased fear of cancer or increased vigilance about prostate cancer screening, leading to more frequent screening and often multiple prostate biopsies. These emotional responses persisted for many years in most of our subjects. A few men were reassured about their persistently elevated PSA, but only after multiple negative prostate biopsies. These data suggest that there should be more effort to develop guidelines for subsequent prostate biopsies after an initial negative biopsy, and more effort to encourage primary care clinicians and urologists to discuss emotional risks of PSA testing with their patients.

\section{Acknowledgements}

This research was supported through a grant from the Department of Defense as part of the Dean and Betty Gallo Prostate Cancer Center (W81XWH-06-1-0514). We thank the Morris Regional Health Partnership and American Cancer Society for their assistance with the study.

\section{Competing Interests}

The authors have no competing interests.

\section{References}

[1] Croswell, J.M., et al., Cumulative incidence of false-positive results in repeated, multimodal cancer screening. Annals of Family Medicine, 2009. 7(3): p. 212-22.

[2] Kilpelainen, T.P., et al., False-positive screening results in the European randomized study of screening for prostate cancer. European Journal of Cancer, 2011. 47(18): p. 2698-705.

[3] Schroder, F.H., et al., Screening and prostate-cancer mortality in a randomized European study. N Engl J Med, 2009. 360(13): p. 1320-8.

[4] Park, J.J., et al., Prostate-specific antigen velocity before and after elimination of factors that can confound the prostate-specific antigen level. Int J Radiat Oncol Biol Phys, 2012. 82(3): p. 121721.

[5] Barton, M.B., et al., Increased patient concern after false-positive mammograms: clinician documentation and subsequent ambulatory visits. Journal of General Internal Medicine, 2001. 16(3): p. 150-6.

[6] Byrne, M.M., et al., Healthcare use after screening for lung cancer. Cancer, 2010.116(20): p. 4793-9.

[7] Kilpelainen, T.P., et al., False-positive screening results in the Finnish prostate cancer screening trial. British Journal of Cancer, 2010. 102(3): p. 469-74.

[8] Seigneurin, A., et al., Association of diagnostic work-up with subsequent attendance in a breast cancer screening program for false-positive cases. Breast Cancer Research \& Treatment, 2011. 127(1): p. 221-8.

[9] Taylor, K.L., et al., Quality of life and trial adherence among participants in the prostate, lung, colorectal, and ovarian cancer screening trial. Journal of the National Cancer Institute, 2004. 96(14): p. 1083-94.

[10] Ford, M.E., et al., Effects of false-positive prostate cancer screening results on subsequent prostate cancer screening behavior Cancer Epidemiology, Biomarkers \& Prevention, 2005. 14(1): p. 190-4.

[11] Aro, A.R., et al., False-positive findings in mammography screening induces short-term distress - breast cancer-specific concern prevails longer. European Journal of Cancer, 2000. 36(9): p. 1089-97.

[12] Brewer, N.T., T. Salz, and S.E. Lillie, Systematic review: the long-term effects of false-positive mammograms. Annals of Internal Medicine, 2007. 146(7): p. 502-10. 
[13] Dobrovoljski, G., et al., False-positive results in neuroblastoma screening: the parents' view. Journal of Pediatric Hematology/Oncology, 2003. 25(1): p. 14-8.

[14] Essink-Bot, M.L., et al., Short-term effects of population-based screening for prostate cancer on health-related quality of life. Journal of the National Cancer Institute, 1998. 90(12): p. 925-31.

[15] Fowler, F.J., Jr., et al., The impact of a suspicious prostate biopsy on patients' emotional, socio-behavioral, and medical care outcomes. Journal of General Internal Medicine, 2006. 21(7): p. 715-21.

[16] Gilbert, F.J., et al., Breast screening: the emotional sequelae of false-positive recall in women with and without a family history of breast cancer. European Journal of Cancer, 1998. 34(13): p. 20104.

[17] Katz, D.A., et al., Health perceptions in patients who undergo screening and workup for prostate cancer. Urology, 2007. 69(2): p 215-20.

[18] Lampic, C., et al., Short- and long-term anxiety and depression in women recalled after breast cancer screening. European Journal of Cancer, 2001. 37(4): p. 463-9.

[19] McGovern, P.M., et al., False-positive cancer screens and healthrelated quality of life. Cancer Nursing, 2004. 27(5): p. 347-52.

[20] Salz, T., A.R. Richman, and N.T. Brewer, Meta-analyses of the effect of false-positive mammograms on generic and specific psychosocial outcomes. Psycho-Oncology, 2010. 19(10): p. 102634 .
[21] van der Steeg, A.F.W., et al., Effect of abnormal screening mammogram on quality of life. British Journal of Surgery, 2011. 98(4): p. 537-42.

[22] Macefield, R.C., et al., Impact of prostate cancer testing: an evaluation of the emotional consequences of a negative biopsy result. British Journal of Cancer, 2010. 102(9): p. 1335-40.

[23] McNaughton-Collins, M., et al., Emotional effects of a suspicious prostate cancer screening test followed by a benign biopsy result. Am J Med, 2004. 117(10): p. 719-25.

[24] Fowler, F.J., Jr., et al., The impact of a suspicious prostate biopsy on patients' emotional, socio-behavioral, and medical care outcomes. J Gen Intern Med, 2006. 21(7): p. 715-21.

[25] Andersen, R.M., Revisiting the behavioral model and access to medical care: does it matter? J Health Soc Behav, 1995. 36(1): p. $1-10$.

[26] Ferrante, J.M., E.K. Shaw, and J.G. Scott, Factors influencing men's decisions regarding prostate cancer screening: a qualitative study. J Community Health, 2011. 36(5): p. 839-44.

[27] Borkan, J.M., Immersion/Crystallization, in Doing Qualitative Research, B.F. Crabtree and W.L. Miller, Editors. 1999, SAGE Publications: Newbury Park. p. 179-194.

[28] Brett, A.S. and R.J. Ablin, Prostate-cancer screening--what the U.S. Preventive Services Task Force left out. N Engl J Med, 2011. 365(21): p. 1949-51.

[29] Moyer, V.A. and U.S.P.S.T. Force, Screening for prostate cancer: U.S. Preventive Services Task Force recommendation statement. Ann Intern Med, 2012. 157(2): p. 120-34. 